\section{USER-FRIENDLY HEAD MOUNTED CAMERA}

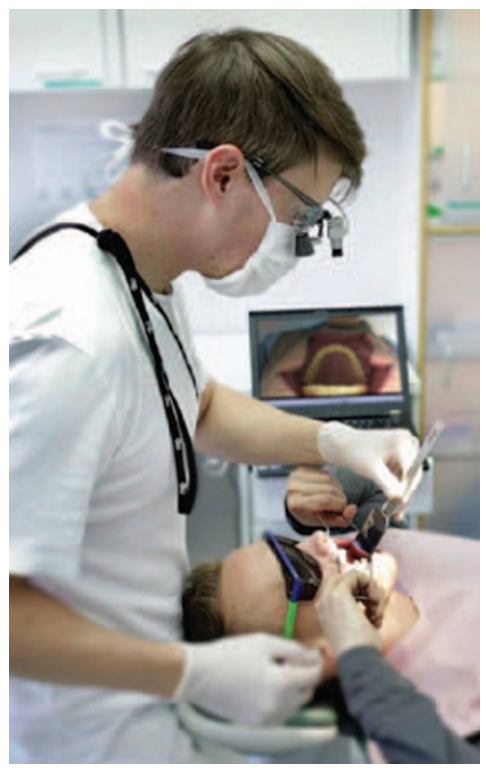

The Futudent HD-camera from Finland is a tiny, head-mounted digital camera with user-friendly software and a cloud-based server, for sharing and storing videos, images and documents.

Engineers developed the Futudent HD-camera to answer the needs of a dental student who wished for an efficient, easy-touse camera that would help him study and share his professional experience with colleagues.

The Futudent HD-camera enables HD video and sound recording from the dentist's point of view as s/he works. The lightweight camera is attached to the dentist's magnifying loupes. The dentist controls the filming with a foot pedal and can mark the most important moments of the procedure with the pedal while working. The software presents all the highlighted parts, so there is no difficulty in finding them in playback. You can attach the camera to your computer with a USB cable.

The videos can be uploaded to the user's private portfolio at the Futudent-server for later viewing and sharing. The server uses bank-level security, enabling a secure and effective channel for dentists to share information.

www.futudent.com

\section{ALL OF YOUR FAVOURITE CONSUMABLES}

Plandent supply all of your favourite infection control consumable products at very competitive prices. The company stocks all leading brands as well as the more cost effective Orbis range, including gloves, face masks, disinfecting liquids and wipes and sterilisation pouches.

Effective sterilisation of hand instruments begins with the Hydrim washer/disinfector from SciCan supplied by Plandent. This simple to operate unit automatically washes, rinses, disinfects and dries your dental instruments at the press of a button. To fully personalise your Hydrim there are a range of accessories to suit your individual practice needs.

Statim autoclaves are available in a range of sizes depending on the capacity you're looking for. These reliable and effective autoclaves process your instruments quickly, reducing instrument investment and increasing efficiency in your practice. Both the Hydrim and Statim are supported by Plandent's accredited nationwide service network.

Plandent's team are fully trained on the latest guidelines in infection control and are kept fully up to date with any changes, enabling them to provide you with current information, guidance and advice.

Call Plandent on 0500500322.

\title{
EVERYTHING FOR IRRIGATION, DEBRIDEMENT AND DISINFECTION
}

QED's cost-effective range of irrigants and related products are extremely useful for every practitioner with an interest in endodontics.

They offer a comprehensive range which includes chlorhexidine $2 \%$ for use as the final irrigation step for long lasting cleaning and disinfection of root canals; sodium hypochlorite $2 \%$ and $<6 \%$ which are used for the irrigation, debridement and disinfection of root canals during and after instrumentation; citric acid which is recommended as a root canal irrigant/cleaner and conditioner - this mild chelating agent can also be used to dissolve calcium hydroxide from canals; EDTA which facilitates the removal of the smear layer and dentine 'mud', enhancing disinfection and preparing the dentine walls for advanced adhesion of filling materials; eucalyptus oil plus many other very useful products.

QED's extremely cost-effective irrigant range is just one of the additions detailed within their endodontic catalogue and featured on www. qedendo.co.uk, the online version.

\section{FROM DESIGN TO INSTALLATION}

When the time comes to refresh the look of your dental practice, it is important to use a company with experience and specialist expertise.

Whether you are completely reinventing the appearance of your practice, or simply updating it, Tavom provide customised furniture and cabinetry of the highest quality.

Tavom's personalised cabinetry is supplied by RPA Dental, the equipment specialists with over 40 years' experience in delivering an unrivalled service and after-sales support.

Their experienced team will guide you through the entire process from design to installation, and you can be sure your new practice will be created with minimal disruption to your daily routine.
Make sure you work with names you can trust and create your ideal practice.

Call Tavom UK on 0870752

1121 or visit www.tavom.com. 